\title{
Wer darf dazugehören? Anstöße Postkolonialer Theologie im Kontext der Migration
}

\author{
Michael Nausner
}

\begin{abstract}
In this chapter on belonging in the context of migration, the author introduces some key aspects of postcolonial theology. He begins with reflecting on the plural and ambiguous aspects of any form of belonging. The realization that otherness is a part of oneself proves to be a precondition for the recognition of the real other. The violent construction of an us-them dichotomy ignores the ambiguous starting point of any formation of identity. The second part of the essay offers a brief sketch of post- and decolonial critique of modernity's exclusions. Finally, acceptance of polyphonic coexistence and multiple belonging in the context of migration is proposed. After all, hybridity of cultural becoming can be detected in much of the biblical material and the history of Christianity.
\end{abstract}

Die Frage der Zugehörigkeit ist eine Frage, die zum Wesen des Menschseins gehört. Der Mensch ist ein Gemeinschaftswesen und ist deshalb abhängig von Zugehörigkeit. In den letzten Jahren ist diese Frage in der Öffentlichkeit wieder neu in den Blick geraten, und zwar vor allem angesichts der steigenden Anzahl von Migrantinnen und Migranten in der Europäischen Union. Die Spannung zwischen nationalstaatlichen Kriterien der Zugehörigkeit und den Kriterien des internationalen Asylrechts und der Genfer Flüchtlingskonvention konkretisiert sich an vielen Einzelschicksalen, aber auch an ganzen Bevölkerungsgruppen. Das problematische Erbe europäischer Staatenbildung, die etwa zeitgleich mit der Entwicklung von Kolonialismus und Imperialismus vonstattenging, wird in dieser Spannung deutlich. Denn die Vorzüge der Staatsbürgerschaft und der Rechtsstaatlichkeit, die die europäischen Staaten sich selbst und einander gewährten, wurden gleichzeitig den eroberten und kolonisierten Gebieten nicht zugestanden. Mit den Auswirkungen dieser problematischen Gleichzeitigkeit leben heute die Menschen Europas, und auf verschiedenen Ebenen gilt es, darum zu ringen, „Zugehörigkeit und Beteiligung jenseits des vertrauten 
Modells nationalstaatlicher Ordnungen zu denken." ${ }^{\text {I }}$ Denn wie sich an den vielerorts erstarkten Nationalismen Europas ablesen lässt, ist die Relativierung des staatlichen Souveränitätsverständnisses durch Immanuel Kant am Ende des 18. Jahrhunderts offensichtlich auch heute noch weniger wirksam als das ältere Westfälische System aus der Mitte des 17. Jahrhunderts, das Recht und Rechtsstaatlichkeit fest innerhalb territorialer Grenzen verankert. ${ }^{2}$ So werden Grenzen heute vielfach strikt als Werkzeuge zur Ausgrenzung verwendet und verabsolutiert, anstatt „die Vielfalt von Grenzphänomenen und Modi des

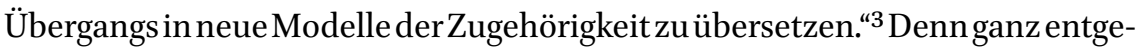
gen der politischen Terminologie der Naturalisierung von Ausländerinnen und Ausländern, sind Nationen nichts Natürliches, und Fragen der Zugehörigkeit müssen immer wieder neu verhandelt werden. ${ }^{4}$ Dabei ist es nicht zuletzt von entscheidender Bedeutung, Menschen nicht auf eine einzige Zugehörigkeit zu reduzieren. Dass eine solche Reduktion eine typische Herrschaftsstrategie ist und oft destruktive Folgen hat, weist Amartya Sen in seiner Studie zu Identität und Gewalt überzeugend auf. Er spricht in dem Zusammenhang vom Problem der „singulären Klassifizierung“ (single classification) ${ }^{5}$ und kritisiert ein solches Verständnis von eindimensionaler Zugehörigkeit etwas ironisch als „zivilisatorische Einkerkerung" (civilizational incarceration). ${ }^{6}$ "Als sozialer Relationsbegriff", schreibt die Sozialethikerin Marianne Heimbach-Steins, „verweist Zugehörigkeit [...] auf verschiedene Wirklichkeitsbereiche: auf familiäre Herkunft und/oder ethnische Abstammung, auf eine soziale Gruppe, in der jemand aktuell lebt; auf die Gesellschaft, in der eine Person sich aufhält und deren Mitglied sie de facto ist; auf den Staat, dessen Staatsangehörigkeit jemand besitzt und in dessen (Rechts-)Ordnung sie beziehungsweise er einge-

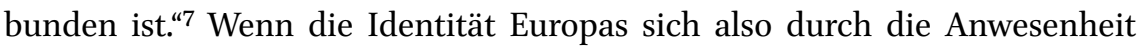
einer wachsenden Anzahl von Migrantinnen und Migranten wandelt, darf diese Situation nicht missverstanden werden als eine Begegnung zwischen mobilen und stabilen Identitäten. Vielmehr zeigt sich hier, was Zugehörigkeit grundsätzlich ausmacht: Sie ist immer eingebettet in eine Vielfalt verschiedener Zugehörigkeiten, deren Eigenart sich nicht aufteilen lässt in solche

1 Marianne Heimbach-Steins, Grenzverläufe gesellschaftlicher Gerechtigkeit. Migration - Zugehörigkeit - Beteiligung, Paderborn 2016, 39.

2 Vgl. a.a.O., $44-45$.

3 A.a.O., 49.

4 Vgl. a.a.O., $5^{2}$.

5 Vgl. Amartya Sen, Identity and Violence. The Illusion of Destiny, London/New York 2013, xvi.

6 Vgl. a.a.O., 10.

7 Heimbach-Steins, Grenzverläufe, 55 . 
diesseits und jenseits nationalstaatlicher Grenzen. Identitäten sind multipel und komplex, und die „Relativierung von Zugehörigkeiten“ könnte deshalb „ein Ansatzpunkt sein, von dem her auch die Frage politischer Zugehörigkeit $[\ldots]$ neu gedacht werden kann."8

\section{Zugehörigkeit als plural und mehrdeutig}

Ich möchte deshalb zunächst über den Umstand reflektieren, dass Zugehörigkeit einerseits nicht als singulär verstanden werden kann (wir gehören immer zu mehr als einer Gruppe bzw. Gemeinschaft gleichzeitig) und andererseits Zugehörigkeit ihrem Wesen nach mehrdeutig ist (nie gehören wir vollständig zu dieser oder jener Gemeinschaft). Viele Menschen ringen mit alltäglichen Gefühlen oder Anfechtungen, wenn sie zwar nach außen hin zu einer Gruppe gehören, innerlich sich jedoch fremd fühlen. Bin ich, die ich eine türkische Mutter habe, eine gute und damit „eigentliche“ Deutsche? Darf ich mich mit meinem lebendigen Interesse für den Islam wirklich als Christ bezeichnen? Wie verändert sich meine familiäre Zugehörigkeit jetzt, da ich einen Partner aus einer gesellschaftlich völlig anders situierten Familie geheiratet habe? Ich glaube nicht, dass solche Zweifel ungewöhnlich oder beklagenswert sind. Vielmehr gehören sie zum Wesen all unserer verschiedenen Zugehörigkeiten. Sie sind ein Zeichen für die Dynamik, die jede Zugehörigkeit auszeichnet und ein Zeichen für die Prozesshaftigkeit, die sowohl individueller als auch kollektiver Identität grundsätzlich innewohnt.

Dass Zugehörigkeit nie ganz stabil und eindeutig ist, hat der Phänomenologe Bernhard Waldenfels auf den Punkt gebracht, wenn er von einer Zugehörigkeit in der Unzugehörigkeit spricht. Eine gewisse „Unzugehörigkeit" ist Teil jeder Zugehörigkeit, denn „wer zu einer Familie, zu einem Volk, zu einem Stand, $\mathrm{zu}$ einer Religionsgemeinschaft oder zu einer Kultur gehört, gehört niemals schlechterdings dazu. Ferne, Abwesenheit, Distanz, auch Momente der Einsamkeit und der Ortlosigkeit [...] gehören zu ihrerEssenz."9 Aufmerksamkeit für diese leise und oft kaum wahrnehmbare Dimension des Fernen und Fremden, die jeder Zugehörigkeit innewohnt, halte ich für entscheidend. Waldenfels, der sich jahrzehntelang mit dem Phänomen des Fremden in all seiner Komplexität auseinandergesetzt hat, ist ein unermüdlicher Mahner für eine solche Aufmerksamkeit. Er ist überzeugt davon, dass niemand sich

8 A.a.O., 56.

9 Bernhard Waldenfels, Grundmotive einer Phänomenologie des Fremden, Frankfurt 2006, 56-57 (meine Hervorhebung). 
je selbst ganz gehört, was daran liegt, dass das Ich ein Anderer ist, „weil die Fremdheit im eigenen Hause beginnt. ${ }^{\text {"10 }}$ Diese Überzeugung von der mehrfachen Zugehörigkeit als Grundmerkmal menschlichen Seins erinnert an Paul Ricoeurs Rede vom Selbst als dem Anderen, die er einem zu sehr von Heidegger geprägten Existentialismus entgegenhielt. Das Selbst lässt sich schlicht nicht trennen vom Anderen: „Das, Ich selbst' ist nicht irgendein Ausdruck, der einem anderen nur gegenübersteht, er ist mit diesem regelrecht verflochten “. ${ }^{11}$

In seinen Reflexionen darüber, was zwischen Kulturen geschieht, ${ }^{12}$ beschreibt Waldenfels, wie das Fremde und Ferne beim Individuum selbst entsteht. „Niemand“, so Waldenfels, „ist seiner Kultur ganz und gar zugehörig“. ${ }^{13}$ Das merkt man schon allein daran, dass jeder Einzelne unter uns seine „Kultur" unterschiedlich beschreibt. Kultur - kulturanthropologisch verstanden - ist ja nicht ein fest umrissenes Phänomen, sondern ein komplexes Netz von Zeichen-, Kommunikations- und Praxissystemen, das per definitionem in ständigem Wandel begriffen ist. So gesehen ist so etwas wie völlige oder totale Zugehörigkeit schlicht und einfach nicht möglich. Was sich über kulturelle Zugehörigkeit sagen lässt, hängt zusammen mit einer grundlegenden existentiellen Fremdheit, die darin zum Ausdruck kommt, dass „niemand je völlig bei sich selbst und in seiner Welt zu Hause“ ist. ${ }^{14}$ Solche Fremdheit, so Waldenfels, betreffe bereits etwas so Grundlegendes wie unseren Rufnamen: „Der sogenannte Eigenname ist ein halbfremder Name, weil er von Anderen stammte. ${ }^{“ 15}$ Wenn also die These von dieser grundlegenden inneren Fremdheit stimmt, dann bedeutet das auch, dass niemand je seine eigene Identität ganz kontrolliert, niemand sich ganz selbst gehört und deshalb auch nie vollständig bestimmen kann, wozu er oder sie gehört. Diese Einsicht hat etwas Beunruhigendes und Verstörendes, aber gleichzeitig, glaube ich, ist sie von positiver Bedeutung für den Umgang mit Fremden. In dieser Öffnung hin zum anderen besteht das Tröstliche an dieser Einsicht, ja ich würde sagen, darin liegt das Friedenspotential dieses Zugeständnisses. Denn, so Waldenfels, die „Fremdheit inmitten meiner selbst öffnet Wege zur Fremdheit des Anderen.“16

\footnotetext{
$10 \quad$ A.a.O., 28.

11 Vgl. Radiosendung zum 100. Geburtstag Paul Ricoeurs: „Paul Ricoeur: Der Weg zum wirklichen Verständnis des Anderen - https://www.swr.de/-/id=10883356/property=download/ nid=660374/s4a19m/swr2-wissen-20130227.pdf, 7 .

12 Vgl. WaLdenfels, Grundmotive, 109-132.

13 A.a.O., 119.

14 A.a.O., 126.

15 A.a.O., 88.

16 A.a.O., 84 .
} 
Die Fremdheit in mir öffnet Wege zur Fremdheit der Anderen. Das ist eine Einsicht, die zunehmend im kulturellen Selbstverständnis der westlichen Welt verdrängt zu werden scheint. Kulturelle Identität wird in der öffentlichen Darstellung verstanden als vollständig, eindeutig, exklusiv und abgegrenzt; kurzum als etwas, das man klar definieren und so kontrollieren kann. Die „Anerkennung der Vielfalt unserer Zugehörigkeiten“, ${ }^{17}$ die Amartya Sen für entscheidend hält für den Frieden in unserer heutigen Welt, spielt in der Öffentlichkeit eine sehr untergeordnete Rolle. Ein solches Verständnis klar umrissener kultureller Identität wird im Zusammenhang mit den Debatten um die Migration deutlich. Das Thema Migration beherrscht in der westlichen Welt - und nicht nur in ihr - die Titelseiten der Tageszeitungen und Internetauftritte wie kaum ein anderes Thema. Hinter vielen der großen und medienwirksamen Berichte lässt sich nach meiner Beobachtung ein verkürztes Verständnis von Zugehörigkeit identifizieren. Das Bauen neuer Mauern, das im Zusammenhang mit der Ankunft einer großen Anzahl von Flüchtlingen im Jahr 2015 neu angefacht wurde, wird vielfach damit begründet, dass europäische Identität geschützt werden müsse. Es gehe um klare Fronten bezüglich der Zugehörigkeit. Auf analoge Weise baut der „Brexit“, also der Ausstieg Großbritanniens aus der Europäischen Union, unter anderem auf die politisch und medial verbreitete Fehlinformation, dass britische Identität unabhängig sei und sich unter Abschottung von der Umwelt besser entfalten könne. Abschottung scheint auch zur Strategie Donald Trumps zu gehören, der trotz oder sogar aufgrund seiner Versprechen, eine kompakte Mauer entlang der US-mexikanischen Grenze hochzuziehen und außerdem keine Muslime mehr ins Land zu lassen, zum Präsidenten der Vereinigten Staaten gewählt wurde. US-amerikanische Identität, so die Hoffnung, werde durch solche Maßnahmen geschützt und gestärkt. Die Liste könnte noch viel länger gemacht werden. Auch in der deutschen Gesellschaft wird immer häufiger der Traum von einer reinen, eindeutigen, von außen nicht gestörten Zugehörigkeit propagiert. Der Preis für solche strikten Grenzziehungen zwischen totaler Zugehörigkeit einerseits und völliger Fremdheit, ja sogar Feindschaft, andererseits ist ein hoher. Denn eine so polarisierende Vorstellung schürt nicht nur die Ressentiments auf der anderen Seite der Grenze, sondern lässt auch die eigene Identität verkümmern, wenn wir Waldenfels' These von der notwendigen Fremdheit der eigenen Identität gelten lassen. 
Postkoloniale Theorie kann hilfreich sein, um in Zeiten wie diesen nicht in die Falle eines polarisierenden Denkens einander ausschließender Kulturen zu geraten. Wenn in der Gesellschaft eine allgemeine Verunsicherung bezüglich kultureller Identität zu spüren ist, scheint es mir wichtig zu sein, den wieder neu auftauchenden Gespenstern einer „reinen“ Kultur, einer überlegenen Zivilisation oder gar einer höheren „Rasse“ ein differenziertes Verständnis von kultureller Zugehörigkeit entgegenzuhalten. Waldenfels ist in gewisser Hinsicht ein Brückenbauer hin zu postkolonialer Analyse, wenn er bezüglich der Dynamik zwischen den Kulturen schreibt: „Am Anfang steht nicht nur die Differenz, sondern auch eine Mischung, die jedes familiäre, nationale, rassische oder kulturelle Reinheitsideal als bloßes Phantasma entlarvt." ${ }^{18}$ Damit betont er etwas, was auch postkoloniale Denkerinnen und Denker verschiedener Couleur meinen, wenn sie darauf hinweisen, dass Kultur von Anfang an und unweigerlich gemischt, ja hybrid ist. ${ }^{19}$ Das ist zunächst einmal nur beschreibend gemeint und nicht ethisch bewertend. Hier wird dem Wesen kultureller Dynamiken nachgespürt. Die Einsicht in die grundlegende Hybridität kulturellen Werdens ist eine wichtige Voraussetzung, um ein verantwortliches Ethos im interkulturellen Kontext zu entwickeln und um faire Modelle im Miteinander der Kulturen zu entwerfen. Eine der Grundeinsichten postkolonialen Denkens ist eben diejenige, dass jede kulturelle Zugehörigkeit komplex ist und deshalb ein Verständnis von Kultur als rein und abgeschlossen notwendigerweise ein Konstrukt darstellt, das in der Geschichte schon viel Unheil angerichtet hat. Gerade auch für die Theologie, die bis heute in vielerlei Weise mit ihrer Prägung durch koloniale Denkweisen und Strukturen ringt, kann diese Einsicht postkolonialer Theorie in die unausweichliche Hybridität kultureller Identität wegweisend sein, denn sie gilt auch ihrer eigenen Identität. „Postkoloniale Theorie und der Paradigmenwechsel in der Kulturanthropologie machen deutlich“, schreibt Judith Gruber, „dass christliche Identität (von Anfang an) plural, fragmentiert und vom Fremden durchzogen ist.“20 Wenn diese Mischung, diese Interkulturalität zum Wesen menschlicher und eo ipso christlicher Identität gehört, dann wird für die Theologie postkoloniale Theorie ein wichtiger Gesprächspartner, denn sie unterminiert auf heilsame Weise den hegemonialen Status und Anspruch einer zeitlosen und monokulturellen

\footnotetext{
18 Waldenfels, Grundmotive, 118.

19 Vgl. Номі К. Внавна, Die Verortung der Kultur, Tübingen 2000.

20 Judith Gruber, Theologie nach dem Cultural Turn. Interkulturalität als theologische Ressource, Stuttgart 2013, 13.
} 
Theologie. ${ }^{21}$ Und wenn postkoloniale Theorie diese Rolle spielen darf, kann sie die christliche Theologie nachhaltig vor einer gewaltsamen Konstruktion der Anderen bewahren.

Der postkoloniale Klassiker schlechthin istEdwardW.Saids Buch Orientalismus, geschrieben in den späten 1970er-Jahren. Said zeigt anhand einer Analyse britischer Literatur des 19. Jahrhunderts, wie der Westen - der Okzident das paradigmatisch Andere - den Orient - nicht etwa durch aufmerksame Begegnung oder kommunikativen Austausch kennengelernt und beschrieben hat, sondern sein Bild vom Orient als das Andere par excellence produziert hat. Hier geht es nicht um rohe Gewalt im militärischen Sinne, sondern um etwas viel schwerer Greifbares und deshalb umso Wirksameres, nämlich um epistemische Gewalt. Sprache beschreibt nicht nur, wie wir spätestens seit Michel Foucault wissen, sondern produziert auch etwas über die Köpfe der Beschriebenen hinweg. Die Wissensproduktion, die im Falle des Orientalismus vor sich geht, ist eine, die das Machtgefälle zwischen Okzident und Orient pflegt und aufrechterhält. Es besteht eine Hegemonie von europäischer Überlegenheit gegenüber orientalischer Rückständigkeit, wie es Said ausdrückt. ${ }^{22}$ Besonders heimtückisch ist das westliche „Wissen“ über den Orient, wenn es sich wissenschaftlich kleidet. Eine wichtige Pointe Saids ist, dass es letztlich keine eingrenzbaren Phänomene gibt, die sich als „Orient" oder „Okzident" definieren ließen. ${ }^{23}$ Beide sind Konstrukte. Deshalb kritisiert er das Ansinnen, man könne über den Orient „objektives Wissen“ erlangen, das unabhängig wäre von den politischen Gegebenheiten. ${ }^{24}$ Das Wissen über den Orient ist eine Konstruktion, die der Aufrechterhaltung einer imaginierten Hegemonie dient. Und somit ist Wissen immer auch politisch.

Saids bleibender Beitrag zum öffentlichen Diskurs im Westen ist seine Analyse der Machtdynamik, die in den sogenannten "Schilderungen“ des Orients zum Ausdruck kommt. Ich glaube, in der Hinsicht gibt es für Kirche, Wissenschaft und Politik auch heute noch viel Nachholbedarf. Wie leicht und wie schnell schleichen sich nicht polarisierende Beschreibungen einer komplexen kulturellen Wirklichkeit in unsere mündliche und schriftliche

\footnotetext{
21 Vgl. a.a.O., 38.

22 Vgl. Edward W. SAID, Orientalism, New York 1978, 7.

23 Vgl. a.a.O., 331.

24 Vgl. a.a.O., 10.
} 
Wissensproduktion! Wie schnell wissen wir nicht Bescheid über das Wesen der Anderen! Gerade in Zeiten, in denen die Migration zu einem Problem stilisiert wird, ${ }^{25}$ das es zu lösen gilt, ist das der Fall. Die Versuchung der klaren Einteilung in ,wir' und ,die Anderen' ist groß, und damit zusammenhängend auch die Versuchung, zu meinen, die Identität der Anderen zu kennen, ohne ihnen begegnet zu sein.

Die englisch-burmesische Migrationsforscherin Bridget Anderson hat ihrer Studie über die Migrationspolitik Großbritanniens den Namen ,Us' and, them: The dangerous politics of immigration control (deutsch: ,Wir' und, sie. Die gefährliche Politik der Migrationskontrolle) gegeben. Sie beschreibt in diesem Buch Polarisierungstendenzen, die nicht nur in Großbritannien, sondern auch anderswo zu beobachten sind. Anderson betont, dass Mobilität nicht eine Ausnahme ist in der Menschheitsgeschichte, sondern von jeher ein integraler Bestandteil menschlichen Zusammenlebens, ${ }^{26}$ und dass der moderne Territorialstaat mit seinem strengen Grenzregime eine verhältnismäßig junge Erfindung ist. Außerdem ist die Entstehung des Territorialstaats, wie eingangs erwähnt, nicht zu trennen von der Entstehung von Imperialismus und Kolonialismus. Auch wenn der moderne Verfassungsstaat auf hehre Ideale der universalen Menschenrechte baut, so stehen kontinuierlich die Bürgerrechte in starker Spannung mit diesen Menschenrechten. ${ }^{27}$ Denn ganz offensichtlich haben Menschen mit und ohne Staatsbürgerschaft konkret sehr unterschiedliche Rechte, auch wenn sie im selben Gemeinwesen leben. Anderson beschreibt die meist begrenzte Reichweite grundlegender Rechte: „Allgemeinheit und Gleichheit, die man zu den Charakteristika heutiger Staatsbürgerschaft in liberalen Demokratien zählt, stellt man sich typischerweise in der Praxis nur innerhalb geschlossener nationaler Gesellschaften vor.“28 Was die Rolle der meisten Migranten im Gemeinwesen europäischer Staaten betrifft, so wird deutlich, dass „Staatsbürgerschaft nicht für Universalismus, sondern für ein Abschließen“29 steht. Auf Dauer ist es jedoch ein großes Problem, wenn

25 Vgl. Zygmunt Baumans letzten Essay, in dem er die Begrifflichkeit „Migrationskrise“ kritisch aufs Korn nimmt. - Zygmunt Bauman, Die Angst vor den anderen. Ein Essay über Migration, Frankfurt 2016, 7 .

26 Vgl. a.a.O., 12.

27 Vgl. Seyla Benhabib, Die Rechte der Anderen, Frankfurt 2008.

28 Bridget Anderson, Us and Them? The Dangerous Politics of Immigration Control, Oxford 2013, 97 (Meine Übersetzung und Hervorhebung).

29 A.a.O., 98. 
innerhalb desselben Gemeinwesens für verschiedene Bevölkerungsgruppen unterschiedliche rechtliche Kriterien bestehen. ${ }^{30}$ Deshalb sollte die Phase, während der Migranten zwar denselben Gesetzen unterstehen wie Staatsbürger, diese jedoch gleichzeitig nicht mitbestimmen können, im Interesse der Allgemeinheit so kurz wie möglich gehalten werden. ${ }^{31}$

Staatliche Souveränität - so sehr sie für eine innere Ordnung notwendig ist - stößt bei der Auseinandersetzung mit Migration immer wieder an ihre Grenzen (buchstäblich und im übertragenen Sinne). Denn wer hat „Aufsicht“ über den Souverän Staat, wenn er sich von medial produzierten Ängsten vor Flüchtlingen steuern lässt? Heute scheint es in Europa wieder völlig akzeptabel zu sein, im Interesse des Gemeinwohls, das oft ausschließlich als relevant innerhalb des Rahmens staatlicher Souveränität interpretiert wird, grundlegende menschliche Ideale eines gerechten Zusammenlebens zu ignorieren. Dabei ist es alles andere als ausgemachte Sache, wie "Gemeinwohl" in einer globalisierten Gesellschaft zu interpretieren ist. „Was auf nationalstaatlicher Ebene als Gemeinwohlbelang erscheint, kann sich im globalen Maßstab als sozial und/oder ökologisch unverträgliches Partikularinteresse erweisen“, ${ }^{32}$ wie Heimbach-Steins festhält. Für ein solch begrenztes Verständnis von "Gemeinwohl“ gibt es in den gegenwärtigen politischen Diskussionen in Europa unzählige Beispiele, und es spiegelt sich in der Rede von der Festung Europa, die umgangssprachlich geworden ist.

Wenn bereits aus der rechtlichen Perspektive des Weltbürgertums die grundsätzliche Zugehörigkeitsgerechtigkeit (vgl. Arendts „Recht, Rechte zu haben") einer von Staaten zu sichernden Verteilungsgerechtigkeit vorgeordnet werden muss, ${ }^{33}$ so lässt sich aus schöpfungstheologischer Perspektive ähnliches sagen, nämlich dass sich eine territoriale Eingrenzung des Gemeinwohls nicht rechtfertigen lässt. Die Schöpfung kennt keine Grenze, die das Wohl der einen vom Wohl der anderen trennen würde bzw. die das Wohl der einen auf Kosten der anderen bewahren müsste. Denn im Grunde zielt das Schöpfungshandeln Gottes darauf ab, allem Geschaffenen einen Ort

30 Zugespitzt hat sich diese Problematik Anfang 2019 im Zusammenhang des militärischen Siegs über Daesh, den „Islamischen Staat“. Die Versuchung, deutsche Staatsbürger, die im Terrorstaat mitgewirkt haben, nicht nach deutschem Recht zu behandeln, ist groß. - Vgl. Helene Bubrowski, Wohin mit den Gotteskriegern des IS?, in: Frankfurter Allgemeine Zeitung, 18.2.2019 - https://www.faz.net/-gq5-9jyrq (abgerufen am 13.3.2019).

31 Vgl. Abizadeh Arash, Geschlossene Grenzen, Menschenrechte und demokratische Legitimation, in: polylog 30 (2013), 5-23, 23. Online: http://www.polylog.net/fileadmin/ docs/polylog/30_thema_Abizadeh.pdf (abgerufen am 12.3.2019).

33 Vgl. Seyla Benhabib zitiert in: a.a.O., 88. 
zu schaffen, an dem es in Frieden, Sicherheit und Wohlergehen leben kann. ${ }^{34}$ Hier geht es dementsprechend um ein grundsätzliches und weitest möglich gefasstes Verständnis von Zugehörigkeit, das sogar über das Menschliche hinausgeht. Ich sehe es als einen Rahmen für jene „Solidarität zwischen Menschen“, wie sie Zygmunt Bauman in seinem allerletzten Buch mit dem Titel Die Angst vor den anderen. Ein Essay über Migration einfordert. ${ }^{35}$ In diesem Sinne halte ich Heimbach-Steins' migrationsethische Vorrangregeln für sinnvoll, die im Sinne eines „Ethos globaler Solidarität" eine grundlegende "Symmetrie zwischen Migranten und Eingesessenen“ aufrechterhalten wollen. Denn darin, so Heimbach-Steins, „konvergiert ein biblisch rückgebundener christlichethischer Ansatz mit philosophischen Traditionen des Kosmopolitismus.“36 Um der destruktiven Dynamik eines hegemonial verstandenen Verhältnisses zwischen uns und den anderen entgegenzuwirken, kann als erste Regel der Vorrang der Menschenwürde und der grundlegenden Menschenrechte vor aller Differenz, ${ }^{37}$ als zweite Regel der Vorrang der einzelnen Person vor der Institution ${ }^{38}$ und schließlich als dritte Regel der Vorrang des Gemeinwohls vor Partikularinteressen ${ }^{39}$ verstanden werden. Migrationsethisch korrespondieren diese drei Regeln mit der Notwendigkeit, erstens, das koloniale Paradigma mit seinen hegemonialen Ansprüchen zu überwinden, zweitens, das Recht des/der Einzelnen auf Zugehörigkeit anzuerkennen und einzufordern und, drittens, die Frage des Gemeinwohls im Sinne der tatsächlich bestehenden mehrfachen Zugehörigkeiten immer wieder neu zu bedenken und zu problematisieren. ${ }^{40}$

Die Grenze zwischen uns und den anderen wird auf diese Weise kontinuierlich verhandelt und nie als gegeben angesehen. Unterschiede und damit Grenzen spielen im vielfältigen Zusammenleben natürlich immer eine Rolle. Durch die Problematisierung der "Natürlichkeit“ eben derselben Grenzen, insofern sie immer auch Konstrukte „asymmetrischer Machtverhältnisse“ sind, bleibt jedoch die Zusammengehörigkeit der einen Menschheitsfamilie im Blick. ${ }^{41}$

34 Vgl. Amélé Adamavi-Aho Ekué/Frank Mathwig/Matthias Zeindler (Hg.), Heimat(en)? Beiträge zu einer Theologie der Migration, Zürich 2017, 25.

BaUman, Angst, 24.

36 Неiмbасн-Steins, Grenzverläufe, 92.

37 Vgl. a.a.O., 78.

38 Vgl. a.a.O., 79 .

39 Vgl. a.a.O., 8 o.

40 Vgl. a.a.O., 83.

41 Vgl. a.a.O., 63. 
Um diesen Blick auf die Einheit der Menschheitsfamilie frei zu halten, braucht es eine kontinuierliche Beschäftigung mit den geschichtlich gewachsenen kolonialen Denkmustern und Strukturen.

\section{Die Schattenseite der Moderne}

Das historische und ideologische Fundament unseres heutigen Gemeinwesens muss immer wieder neu geprüft werden, und damit auch die lang bestehenden Verbindungen und Verstrickungen zwischen dem (post)modernen Europa und den scheinbar rückständigen Teilen der Welt. So haben hellhörige Kulturkritiker immer wieder darauf hingewiesen, dass die westliche Moderne bei all ihren Errungenschaften auch eine destruktive Seite hat. Dass diese negative Seite der Moderne verdrängt und vergessen wird oder einfach unbeachtet bleibt, halte ich für einen Hauptgrund dafür, dass heute der Umgang mit migrierenden Menschen oft so problematisch ist. Denn, so der politische Philosoph Achille Mbembe, der aus der ehemaligen deutschen Kolonie Kamerun stammt, dem europäischen Denken wohnte immer schon eine Tendenz inne, „Identität nicht im Sinne gemeinsamer Zugehörigkeit zu ein und derselben Welt zu verstehen, sondern im Sinne eines selbstbezüglichen Verhältnisses “42, das eine wirkliche Einbeziehung anderer in sein Denken verunmöglicht.

Auf ähnliche Weise schildert der Kulturanthropologe Walter Mignolo die Begrenzung der westlichen Moderne konsequent und auf für Europa schmerzliche Weise. Seit Jahrzehnten gehört er jenem Kollektiv an, das sich dafür entschieden hat, nie von der Moderne zu sprechen, ohne sie durch einen Schrägstrich mit Kolonialismus zu verbinden. Im Spanischen wird daraus dann „modernidad/colonialidad“, also Moderne/Kolonialismus. Mit dieser Verknüpfung zweier scheinbar unvereinbarer Begriffe wird zunächst schlicht und einfach auf die historische Tatsache hingewiesen, dass die Entwicklung des Humanismus und der hohen Ideen der Aufklärung geschichtlich einherging mit der Unterdrückung und Versklavung eines Teils der Menschheit. „Kolonialität“ versteht deshalb Mignolo als „die andere und unausweichliche Seite der Modernität ${ }^{433}$. Der in der Moderne angestrebte Universalismus wurde selektiv angewendet, und bis heute verstehen die meisten Europäer unter Universalismus unverkürzt die Ideale der europäischen Aufklärung. Mignolo meint nun, dass verengte Sichtweise nicht eine unerfreuliche

42 Achille Mbembe, Kritik der schwarzen Vernunft, Berlin 2014, 11.

43 Walter D. Mignolo, Local Histories/Global Designs. Coloniality, Subaltern Knowledges, and Border Thinking, Princeton (NJ) 2000, 22. 
Nebenerscheinung der Moderne ist, sondern dass sie eine Wurzel in ihrer denkerischen Begrenzung hat. Er schlägt aber nicht im klassischen marxistischen Stil eine Revolution vor, die letztlich wieder in einem polarisierenden Denken steckenbleibt. Stattdessen befürwortet er in postkolonialem Stil ein Grenzdenken, das sich seiner Blindheit ohne den Beitrag der Anderen stets bewusst ist. Es geht ihm dabei um die Überwindung der strikten Trennung zwischen Subjekt und Objekt des Wissens, weil in dieser Trennung oft ein koloniales, hierarchisches Muster zum Ausdruck kommt. Stattdessen will er einem Wissen auf der Spur bleiben, das in der Begegnung entsteht und also nicht nur auf der einen Seite der „epistemologischen Trennlinie“ entstehen könne. Stattdessen fragt ein Grenzdenken à la Mignolo danach, wie Wissen im Zwischenraum entstehen kann. ${ }^{44}$ Ein solches Wissen bezieht verschiedene Perspektiven nicht als Akt der Großzügigkeit ein, sondern als notwendige Bedingung für ein angemessenes Verständnis kultureller Komplexität. ${ }^{45}$

Ich sehe in diesem Ansatz eine vielversprechende Weise, um Zugehörigkeit im interkulturellen Kontext zu denken und zu praktizieren. Denn in einem solchen Grenzdenken wird unmittelbar klar, dass meine Zugehörigkeit abhängig ist von anders verstandenen Zugehörigkeiten, um konstruktiv zu bleiben. Es bedeutet, dass ich meine eigene Zugehörigkeit gar nicht angemessen verstehen kann, ohne die Perspektive einer anderen Zugehörigkeit wahr- und ernstgenommen zu haben. Und es bedeutet - um noch einmal die Begrifflichkeit von Waldenfels aufzunehmen -, dass meine Zugehörigkeit durchkreuzt bleiben muss von einer Unzugehörigkeit, vom Fremden von außen, um das Fremde im Inneren nicht in Vergessenheit geraten zu lassen.

Aber man muss nicht unbedingt in Walter Mignolos Lateinamerika gehen, um dieser Kritik gegenüber einem eingleisigen Verständnis der Moderne zu begegnen. Nach meinem Dafürhalten ist Walter Benjamin einer der europäischen Denker, der im moralisch kollabierenden Europa eine der Wurzeln für die Katastrophe klar benannt hat, nämlich diejenige, die in der Ambivalenz kultureller Errungenschaften liegt. Ganz knapp zusammengefasst kann man das auf seinem Grabstein lesen: „Jedes Produkt der Kultur ist gleichzeitig auch

44 Vgl. a.a.O., 17-18.

45 Im von Walter D. Mignolo und Catherine E. Walsh gemeinsam verfassten Buch über Dekolonialität (eine Terminologie, die in einem komplexen Verhältnis zur Postkolonialität steht und vor allem von lateinamerikanischen Denkerinnen und Denkern verwendet wird) wird globale Relationalität als Relativierung eindeutiger Zugehörigkeiten theoretisiert. - Walter D. Mignolo/Catherine E. Walsh, On Decoloniality. Concepts, Analytics, Practice, Durham (NC) 2018. 
ein Produkt der Barbarei.“46 Dieser Satz stammt aus seinem letzten Werk Über den Begriff der Geschichte, das er auf der Flucht vor den Nationalsozialisten schrieb, bevor er sich im September 1940 das Leben nahm. Dieses Vermächtnis, so könnte man sagen, trägt Walter Mignolo weiter, wenn er für ein Grenzdenken wirbt.

Auch die 1998 gegründete Wiener philosophische Zeitschrift polylog hat eine Zielsetzung, die ein Denken in diesem Geiste fördern will. Sie ist eine Quelle von Texten zu postkolonialer Theorie im weitesten Sinne. ${ }^{47}$ In der Zielsetzung der Zeitschrift ist von der Bemühung die Rede, „die vielen philosophierenden Stimmen im Kontext ihrer jeweiligen Kulturen vernehmbar und in einer gemeinsamen und gleichberechtigten Auseinandersetzung füreinander fruchtbar zu machen“. Geltungsansprüche, so die programmatische Erklärung, müssen „sich erst interkulturell bewähren“, weshalb ein „Abgehen von einer individuellen, monokulturellen, häufig ethnozentrischen Philosophieproduktion verlangt“ wird. Stattdessen wird eine „dialogische, prozesshafte und grundsätzlich offene Polyphonie der Kulturen und Disziplinen“ angestrebt. ${ }^{48}$

\section{Polyphonie des Zusammenlebens}

Die Metapher der Polyphonie halte ich in unserem globalisierten Zeitalter für besonders sinnvoll, denn sie macht deutlich, dass Zugehörigkeit nicht hermetisch verstanden werden kann. In einem Chor von Kulturen und Disziplinen mitzuschwingen, zeugt von einer Zugehörigkeit, die sich einerseits selbst nicht verleugnet, sich andererseits aber auch dessen bewusst ist, dass der Klang der eigenen Identität ohne die Resonanz anderer Identitäten nicht zu voller Blüte gelangen kann. ${ }^{49}$ Meine eigene (kulturelle) Stimme bekommt erst in der Resonanz und gegebenenfalls auch Dissonanz mit anderen (kulturellen) Stimmen ihre charakteristische Färbung. Die musikalische Metapher

46 Walter Benjamin, Illuminationen. Ausgewählte Schriften 1, Frankfurt 1977, 251-261, 254 .

47 Vgl. u.a.: polylog 4 (1999): Frau und Kultur. Kolonisierung von Differenz, in: http:// www.polylog.net/aktuelles-heft/polylog-4/ (abgerufen am 12.3.2019) - polylog 8 (2001): Hybridität, in: http://www.polylog.net/aktuelles-heft/polylog-8/ (abgerufen am 12.3.2019). polylog-Zielsetzung, in: http://prof.polylog.org/obj-de.htm (abgerufen am 12.3.2019) Für die systematische Theologie wünsche ich mir noch eine Zeitschrift mit einer solchen Zielsetzung. Die interdisziplinäre katholische Zeitschrift concilium kommt dieser Zielsetzung in der Praxis recht nahe.

49 Vgl. auch Hartmut Rosa, Resonanz. Eine Soziologie der Weltbeziehung, Berlin 2016. Rosa vermeidet es allerdings leider fast vollständig, in seinem großen Werk Resonanz bzw. Dissonanz zwischen den Kulturen zu behandeln. 
beleuchtet die Tatsache, dass für ein wirkliches Verstehen alle Stimmen gehört werden müssen, gerade auch die Stimmen, denen traditionellerweise kein Gehör geschenkt worden ist. Wenn dauerhaft Stimmen verdrängt werden, dann ist die planetarische Polyphonie beeinträchtigt. Mit dem Begriff "planetarisch" will ich hier nur andeuten, dass die Verteidigung der Vielfalt der Stimmen, die postkoloniale Theorie auf der Ebene der Kultur praktiziert, nicht getrennt werden darf von der Wahrnehmung der Stimmen der Tierwelt und der ökologischen Welt insgesamt. ${ }^{50}$ Eine solche Zusammenschau der verschiedenen Sphären der einen planetarischen Lebenswelt bietet Catherine Keller. In Zeiten des Anthropozän geht sie von einem weit gefassten Kollektiv aus, in das die Menschheit als organischer Teil eingebettet ist. In ihrem Buch Political Theology of the Earth beschreibt sie eine planetarische Öffentlichkeit, in der unterdrückte Menschen gemeinsam mit der geschundenen Schöpfung Allianzen eingehen. ${ }^{51}$

\section{$8 \quad$ Postkolonialität vor Ort}

Bevor ich mit einigen Reflexionen darüber schließe, was eine postkolonial inspirierte Theologie im Kontext der Migration beizutragen hat, will ich noch einen kurzen Blick in die koloniale Vergangenheit Deutschlands werfen. Es hat sich in den letzten Jahren diesbezüglich einiges getan. Das kann man paradigmatisch an den beiden Versionen der Einführung in postkoloniale Theorie von María do Mar Castro Varela und Nikita Dhawan ablesen. Haben die Verfasserinnen in der ersten Ausgabe dieses Werkes von 2005 noch darüber geklagt, dass in Deutschland die prägende Rolle des Kolonialismus kaum in der Öffentlichkeit wahrgenommen wird, ${ }^{52}$ weisen sie in der zweiten Auflage von 2015 darauf hin, dass „in den letzten Jahren eine breite Auseinandersetzung mit dem deutschen Kolonialismus eingesetzt ${ }^{“ 53}$ hat. Das mag unter anderem mit dem Gedenken an die gut hundert Jahre zurückliegende Ausrottung der Herero und Nama zu tun haben, die Jürgen Zimmerer als den ersten Genozid des

50 Vgl. Stephen D. Moore, Mayra Rivera (Hg.), Planetary Loves. Spivak, Postcoloniality, and Theology, New York 2010.

51 Vgl. Catherine Keller, Political Theology of the Earth. Our Planetary Emergency and the Struggle for a New Public, New York (NY) 2018, 33 u.ö.

52 María do Mar Castro Varela/Nikita Dhawan, Postkoloniale Theorie. Eine kritische Einführung, Frankfurt 2005, $7 \mathrm{ff}$.

53 María do Mar Castro Varela/Nikita Dhawan, Postkoloniale Theorie. Eine kritische Einführung, 2., komplett überarbeitete und erweiterte Auflage, Bielefeld 2015, 28. 
20. Jahrhunderts bezeichnet hat. ${ }^{54}$ Auf jeden Fall scheinen heute mehr Menschen in Deutschland sich der Tatsache bewusst zu sein, dass das Deutsche Reich eine Schlüsselrolle in den kolonialistischen Bestrebungen des ausgehenden 19. Jahrhunderts gespielt hat und dass die rassistischen Diskurse, die damals gepflegt wurden, Vorboten der nationalsozialistischen „Rassenpolitik“ waren. ${ }^{55}$ Aufgearbeitet ist damit allerdings dieses Kapitel deutscher Geschichte noch lange nicht. Nur ein Beispiel: Das vorliegende Kapitel wurde zunächst als Vortrag an der Carl-von-Ossietzky-Universität in Oldenburg konzipiert. An besagter Universität hat die Historikerin Yvonne Robel ein Geschichtsprojekt betrieben, bei dem es um die Suche nach Spuren des Kolonialismus in Oldenburg ging. Eine solche Spur ist der Heldengedenkstein für Kanonier Kleen, einen Teilnehmer am Genozid (auf dem Gedenkstein „Feldzug“ genannt) an den Herero und Nama am Anfang des vorigen Jahrhunderts. Robel weist im Zusammenhang damit auf einen Umstand hin, den ich für entscheidend halte, nämlich, dass „in erinnerungskulturellen Debatten [...] postkoloniale Ansätze fast immer hinter die NS-Geschichte zurück[treten]. ${ }^{46}$ Das heißt, die nationalsozialistische Vergangenheit ist umfassend aufgearbeitet worden, ihre ideologische Verwurzelung im deutschen Kolonialismus hingegen wird nach wie vor kaum thematisiert. Die Schwierigkeit vieler Migranten, sich heute in der deutschen Gesellschaft zurechtzufinden, mag unter anderem auch mit dem unbewussten Fortbestehen kolonialer Denkweisen in der deutschen Kultur zu tun haben. ${ }^{57}$

\section{$9 \quad$ Postkoloniale Theologie im Kontext der Migration}

Die Theologie hat sich postkolonialer Theorie nur zögerlich geöffnet. Zunächst waren es die Bibelwissenschaften in der angelsächsischen Welt, die sich in den 199oer-Jahren postkolonialer Zugänge bedienten, um „die alten imperialen Kontexte der biblischen Texte“ zu untersuchen, aber auch, um einen neuen Blick auf das „Schicksal dieser Texte in westlichen kolonialen Abenteuern neu zu bedenken. ${ }^{458}$ Erstaunliche neue Leseweisen der Texte ergaben sich nicht zuletzt aufgrund der Analogien zwischen imperialen Verhältnissen der Antike und heutigem Imperialismus. Es folgten theologie- und

\footnotetext{
54 Vgl. a.a.O., 31.

55 Vgl. a.a.O., 29-30.

$5^{6}$ Zitiert in: Maik Nolte, Des Kaisers Kanonier, in: http://www.taz.de/!5082648/ (abgerufen am 12.03.2019).

57 Vgl. Мвемве, Kritik.

$5^{8}$ Moore/Rivera, Loves, 4-5 (Meine Übersetzung).
} 
missionsgeschichtliche Analysen, die die koloniale Ideologie hinter vielen missionarischen Unternehmungen kritisch unter die Lupe nahmen, ${ }^{59}$ sowie Studien, die anhand postkolonialer Theorie die quasi-religiöse Legitimation imperialer Bestrebungen der USA anprangern. ${ }^{60}$ Schließlich gibt es eine wachsende Anzahl von Werken, die versuchen, die Einsichten postkolonialer Theorie für klassische Themen der Systematischen Theologie fruchtbar zu machen. ${ }^{61}$ Es sind die letztgenannten Ansätze, die ich als Beispiele postkolonialer Theologie im eigentlichen Sinne verstehe.

Worin nun besteht die Herausforderung der Migration für die Theologie? Und was hat das postkoloniale Paradigma hier beizutragen? Ich will versuchen, auf diese Fragen unter dem Aspekt der Zugehörigkeit einzugehen. Ich gehe in zwei Schritten vor. Zunächst frage ich nach der grundlegenden Bedeutung von Migration für die Theologie, sodann danach, wie ein postkolonialer Blick auf die Migration die Analyse schärfen kann.

Die Frage der theologischen Bedeutung der Migration bewegt mich spätestens seit einer Konferenz zum Thema Migration als Zeichen der Zeit an der theologischen Fakultät der Universität Salzburg im Jahre 2012. Seit damals nehme ich mit zunehmendem Interesse und Erstaunen wahr, dass Migration nicht nur eine grundlegende conditio humana ist, sondern auch aus dem erzählerischen Erbe der christlich-jüdischen Tradition nicht wegzudenken ist. Das bedeutet auch, dass einerseits christliche Theologie aus ihren eigenen Quellen heraus Perspektiven zur heutigen Migration entwickeln kann und muss, und dass andererseits auch die Realität der Migration eine kritische Perspektive auf die Praxis des Theologisierens erlaubt. ${ }^{62}$ Migration ist ein nicht wegzudenkender Kontext biblischen Materials. Wenn ich mich dem biblischen Material mit

59 Vgl. a.a.O., 6.

6o Vgl. a.a.O., 7 .

61 Ein frühes Beispiel dafür ist: Catherine Keller/Michael Nausner/Mayra Rivera (Hg.), Postcolonial Theologies. Divinity and Empire, St. Louis (MO) 2004. - Für eine Übersicht siehe: Michael Nausner, Postkoloniale Theologien, in: VF 57 (2012), 117-131. - Für den deutschsprachigen Raum wegweisend sind: ANDrEAS NeHRING/ Simon Tielisch (Hg.), Postkoloniale Theologien. Bibelhermeneutische und kulturwissenschaftliche Beiträge, Stuttgart 2013; Andreas Nehring/Simon Wiesgickl (Hg.), Postkoloniale Theologien II. Perspektiven aus dem deutschsprachigen Raum, Stuttgart 2017.

62 Vgl. Judith Gruber/Sigrid Rettenbacher (Hg.), Migration as a Sign of the Times, Leiden 2015, 2. 
der Frage nähere, wer migriert, dann beginnt es spätestens mit dem Aufbruch Abrams ins Ungewisse allein aus Glauben. Er steht als Migrant paradigmatisch für alle späteren Generationen jüdisch-christlichen Glaubens. Es folgen in den hebräischen Schriften zahlreiche Erzählungen von Flucht und Migration aufgrund von Unterdrückung, Hungersnot und Krieg, und in ihnen allen wird die gnädige Gegenwart Gottes bezeugt. Dabei nimmt der Exodus des Volkes Israel aus der Knechtschaft in Ägypten einen zentralen Platz ein. Er prägt „die Ethik des biblischen Israel grundlegend“, und es bündeln sich hier "zentrale Migrationsmotive“63 wie Ausgrenzung in der Fremde und erzwungene Migration etc. Der Israel aufgetragenen Erinnerung an die eigene Fremdheitserfahrung entspricht das Ethos der Fremdenliebe, wie es uns in Lev 19 begegnet. Hier wird der Zugehörigkeitsraum erweitert und „die geltenden Vorstellungen, wer ,wir' sind, grundlegend verändert". Im Spiegel der Fremden wächst das Bewusstsein, „dass auch die eigene Identität ein Moment der Fremdheit birgt." ${ }^{4}$

Das Zeugnis vom Leben Jesu Christi und von der entstehenden Kirche ist auf eine andere, aber verwandte Weise geprägt von einer Mobilität, von Flucht und Wanderungen verschiedener Art. Meistens waren es nicht die sesshaften Menschen vor Ort, die die Autorität bezüglich der Zugehörigkeit zur christlichen Gemeinschaft hatten. Vielmehr wurden die Sesshaften von Wanderpredigern wie Jesus und später Paulus in ihren Kategorien der Zugehörigkeit herausgefordert. Bei Jesus zeigt sich das zum Beispiel darin, dass er in der zentralen Erzählung zur Nächstenliebe einen Fremden diese Nächstenliebe praktizieren lässt (Lk 10,25-37) ${ }^{65}$ Und bei Paulus handelt es sich um das ständige Ringen, auch Heiden in den Bund Gottes mit Israel mit einzubeziehen und so den Zugehörigkeitsraum zu erweitern. Der konkrete Kontext der Migration könnte so gesehen als natürlicher Rahmen für eine christliche Spiritualität verstanden werden, die das Leben der christlichen Gemeinschaft als ein Leben als Gast auf Erden versteht. Die Bindung an Gott, fasst es Heimbach-Steins zusammen, impliziert eine „Fremdheitserfahrung in der Welt،“66 Paradigmatisch für ein solches Selbstverständnis der frühen Christen mag der anonyme Brief an Diognet aus dem 2. Jahrhundert sein, in dem es heißt: Christen „bewohnen jeder sein Vaterland, aber nur wie Beisassen; sie beteiligen sich an allem wie Bürger und lassen sich alles gefallen wie Fremde; jede Fremde ist ihnen Vaterland und jedes

63 Heimbach-Steins, Grenzverläufe, 64.

64 Vgl. a.a.O., 66. - Vgl. auch WALDENFELs, Grundmotive, 28.

65 Vgl. a.a.O., 67 .

66 Vgl. a.a.O., 68. 
Vaterland eine Fremde“67. Ich lese dieses Zitat als ein Zeugnis für ein dynamisches Verständnis christlicher Zugehörigkeit. Zugehörigkeit kann hier schon allein deshalb nicht als ausschließlich missverstanden werden, weil sie von einer grundlegenden und bleibenden doppelten Loyalität gekennzeichnet ist.

Migration und damit zusammenhängend eine gewisse existentielle Heimatlosigkeit sind also ein derart durchgängiges Thema in der Bibel und der Geschichte des Christentums, dass man sich mit Recht fragen könnte, ob aus christlicher Perspektive der epistemologische Blick der Migrierenden nicht oftmals ein schärferer sein müsste als derjenige der sesshaften christlichen Gemeinschaften. Natürlich gehört auch die berühmte konstantinische Wende und die damit zusammenhängende Institutionalisierung und geographische Etablierung und Abgrenzung zum christlichen Erbe, ebenso das zunächst befriedende, gleichzeitig jedoch auch exkludierende cuius regio eius religio des Augsburger Religionsfriedens (1555) und später des Westfälischen Friedens (1648). Aber diese späteren geographischen Eingrenzungen christlicher Gemeinschaft und auch ein gewisses christliches Besitzstandsdenken wie etwa die Rede vom „christlichen Europa“ dürfen nicht über den Umstand hinwegtäuschen, dass die formative Phase der jüdisch-christlichen Tradition von Migration gekennzeichnet ist. Der Kontext entscheidender Einsichten über das Wesen Gottes selbst ist so überwältigend häufig der der Vertreibung, der Flucht, des Wanderns, des Exils und der Diaspora, dass es eigentlich für alle Zeiten eindeutig sein müsste, dass christlicher Gemeinschaft ein entscheidender Verstehenshorizont fehlt, wenn sie sich nicht kontinuierlich und entschieden der Lebenswirklichkeit von Migrantinnen und Migranten öffnet. ${ }^{68}$

\section{1 Zugehörigkeit Migrierender postkolonial betrachtet}

Wenn also nach einem theologischen Verständnis der Migration und des Fremden gefragt wird, dann erweist sich, dass eine verantwortungsvolle

67 Brief an Diognet, вKv, Fribourg 2008. - http://www.unifr.ch/bkv/kapitel79-4.htm (abgerufen 17.6.2016).

68 Zur epistemologischen Bedeutung von Migration für die „katholische“ Identität der Kirche vgl. den Artikel Regina PolaK, Migration und Katholizität, in: Dies./Wolfram REIss (Hg.), Religion im Wandel. Transformation religiöser Gemeinschaften in Europa durch Migration, Interdisziplinäre Perspektiven, Religion and Transformation in Contemporary European Society 9, Wien 2015, 229-292. - Vgl. außerdem MiCHAEL NAUSNER, Kulturelle Grenzerfahrung und die methodistische Konnexio, in: MiCHAEL NAUSNER (Hg.), Kirchliches Leben in methodistischer Tradition. Perspektiven aus drei Kontinenten, RThS 5 , Göttingen 2010, 273-295. 
Sichtweise nur dann entstehen kann, wenn die eigenen Wurzeln in der Migration bzw. auch - um mit Bernhard Waldenfels zu sprechen - die eigene Fremdheit wahrgenommen und anerkannt wird. Diese Fremdheit im eigenen Selbst kann auf unterschiedliche Weise verstanden werden, existentiell, kulturell, geschichtlich etc. Dass eine solche Sichtweise, eine solche Bejahung der eigenen Fremdheit tief verwurzelt ist in der jüdisch-christlichen Tradition, kommt in den Regeln zur Heiligung des alltäglichen Lebens zum Ausdruck. Beispielhaft dafür mag Lev 19,34 stehen: Der Fremdling „soll bei euch wohnen wie ein Einheimischer unter euch, und du sollst ihn lieben wie dich selbst; denn ihr seid auch Fremdlinge gewesen in Ägyptenland“. Eine erstaunliche Programmerklärung, die die Frage der Zugehörigkeit nicht von der Willkür der Mächtigeren, der Besitzenden abhängig macht und so ein Machtgefälle ${ }^{69}$ und somit auch eine Polarisierung aufrechterhält, sondern auf die intime Zusammengehörigkeit Einheimischer und Fremder verweist. Und ganz im Waldenfels'schen Sinne wird diese Zusammengehörigkeit erst deutlich, wenn das Fremde in mir und die eigenen Wurzeln in der Fremde, wahrgenommen und anerkannt werden.

Postkoloniale Theorie kann dazu beitragen, diese theologisch-ethische Herausforderung der Begegnung mit und der Gastfreundschaft gegenüber Fremden ein wenig besser in ihrer ganzen Komplexität zu verstehen. Zunächst kann uns die vor allem von Homi K. Bhabha so ausführlich beschriebene Hybridität allen kulturellen Werdens davor bewahren, die Begegnung mit Migranten als eine oppositionelle misszuverstehen. Denn weder kulturell, noch ökonomisch, noch sozial kommen Migranten aus einer von uns getrennten Welt, auch wenn in den Medien die Darstellungsweise einer „von außen“ kommenden Gefahr überwiegt. Postkoloniale Theorie stellt Werkzeuge zur Verfügung, um die intime Verstrickung wahrzunehmen, die etwa zwischen Afrika und Europa besteht. Jahrhunderte von Kolonisierung und viele Jahrzehnte neokolonialer Ausbeutung verunmöglichen es, die ankommenden Flüchtlinge als Bittsteller von außen abzutun. Vielmehr wird nun das sichtbarer, was schon eine sehr lange Zeit der Fall war: Afrika und Europa, der Okzident und der Orient sind miteinander intim verwoben, wobei das Machtgefälle bisher kontinuierlich Europa am längeren Hebel hat sitzen lassen. Koloniale Verhältnisse leben auf verschiedenen Ebenen weiter.

Zudem kann postkoloniale Theorie das Mandat der christlichen Fremdenliebe insofern verschärfen, als sie die Bedeutung der Begegnung mit dem Fremden nicht als eine mögliche Zusatzübung versteht, sondern als den

69 Heimbach-Steins spricht von „asymmetrischen Machtverhältnissen“. Vgl. НегмваснSTEINS, Grenzverläufe, 63 . 
Ort par excellence, an dem Identitätswerdung stattfindet. An der Grenze zum Fremden hört nicht etwa unsere Identität auf. Vielmehr wird erst an dieser Grenze die eigene Identität verhandelt und sichtbar. Man könnte auch sagen, im Grenzraum zum Fremden hin erweist sich unser Eigenstes. Hier wird Identität verhandelt, um das in postkolonialer Sprache auszudrücken. ${ }^{70}$ Jacques Derrida beschreibt, warum er in der kulturellen Begegnung "Verhandeln“ als einen angemesseneren Begriff als „Dialog“ erachtet. Verhandeln umfasst viel mehr als den sprachlichen Austausch. Der Begriff „Dialog“ steht in der Gefahr, eine Kommunikationsebene auf Augenhöhe vorauszusetzen, die es in der Wirklichkeit kaum je gibt. Verhandeln im Sinne Derridas hingegen verneint das unvermeidliche Machtgefälle nicht, das in jeder interkulturellen Begegnung gegeben ist. ${ }^{71}$ Ich sehe in dieser Vorstellung von einer Verhandlung im Grenzraum eine säkulare Beschreibung des Kontextes, in dem das christliche Gebot der Nächsten-, ja der Feindesliebe gelebt wird. Hier wird nicht vorausgesetzt, wer letztlich drinnen oder draußen ist, sondern im Bewusstsein einer Zugehörigkeit, die immer den konkreten kulturellen Rahmen sprengt, im Grenzraum verhandelt, wie Zugehörigkeit immer wieder neu gelebt werden kann.

Ein christliches Verständnis von Zugehörigkeit setzt per definitionem nicht voraus, wer dazugehört und wer nicht, sondern lässt Zugehörigkeit in der Verhandlung im Grenzraum entstehen, weil christliche Identität entscheidend sich erst dort verwirklicht, wo Fremden begegnet wird.

\section{Bibliographie}

Anderson, Bridget, Us and Them? The Dangerous Politics of Immigration Control, Oxford 2013.

Arash, Abizadeh, Geschlossene Grenzen, Menschenrechte und demokratische Legitimation, in: polylog 30 (2013), in: http://www.polylog.net/fileadmin/docs/ polylog/30_thema_Abizadeh.pdf.

BAuman, ZYGmunT, Die Angst vor den anderen. Ein Essay über Migration, Frankfurt 2016.

Benhabib, Seyla, Die Rechte der Anderen, Frankfurt 2008.

70 Vgl. Michael Nausner, Subjects In-Between. A Theological Boundary Hermeneutic, PhD. Dissertation, Drew University Madison (NJ) 2005.

71 Vgl. JacQues Derrida, Negotiations. Interventions and Interviews, 1971-2001, Stanford 2002. 
Benjamin, Walter, Illuminationen. Ausgewählte Schriften 1, Frankfurt 1977.

Внав на, Номі K., Die Verortung der Kultur, Tübingen 2000.

Der Brief an Diognet, in: Frühchristliche Apologeten und Märtyrerakten aus dem Griechischen und Lateinischen übersetzt, übersetzt von Gerhard Rauschen, Bd. 1, BKV 12, Kempten/München 1913, 157-173. - http://www.unifr.ch/bkv/kapitel79-4 .htm.

Bubrowski, Helene, Wohin mit den Gotteskriegern des IS?, in: Frankfurter Allgemeine Zeitung, 18.2.2019. - https://www.faz.net/-gq5-9jyrq.

Castro Varela, María do Mar/Nikita Dhawan, Postkoloniale Theorie. Eine kritische Einführung, Frankfurt 2005.

Castro Vare la, María do Mar/Nikita Dhawan, Postkoloniale Theorie. Eine kritische Einführung, 2., komplett überarbeitete und erweiterte Auflage, Bielefeld 2015.

DerRIDA, JACQUEs, Negotiations. Interventions and Interviews, 1971-2001, Stanford 2002.

Ekué, Amélé Adamavi-Aho/Frank Mathwig/Matthias Zeindler (Hg.), Heimate(n)? Beiträge zu einer Theologie der Migration, Zürich 2017.

Gruber, Judith, Theologie nach dem Cultural Turn. Interkulturalität als theologische Ressource, Stuttgart 2013.

Gruber, Judith /Sigrid Rettenbacher (Hg.), Migration as a Sign of the Times, Leiden 2015.

Heimbach-Steins, Marianne, Grenzverläufe gesellschaftlicher Gerechtigkeit. Migration - Zugehörigkeit - Beteiligung, Paderborn 2016.

Keller, Catherine/Michael Nausner/Mayra Rivera (Hg.), Postcolonial Theologies. Divinity and Empire, St. Louis (MO) 2004.

Keller, Catherine, Political Theology of the Earth. Our Planetary Emergency and the Struggle for a New Public, New York (NY) 2018.

Мвемве, Achille, Kritik der schwarzen Vernunft, Berlin 2014.

Mignolo, Walter D., Local Histories/Global Designs. Coloniality, Subaltern Knowledges, and Border Thinking, Princeton (NJ) 2000.

Mignolo, Walter D./Catherine E. Walsh, On Decoloniality. Concepts, Analytics, Practice, Durham (NC) 2018.

Moore, Stephen D., Mayra Rivera (Hg.), Planetary Loves. Spivak, Postcoloniality, and Theology, New York 2010.

Nausner, Michael, Subjects In-Between. A Theological Boundary Hermeneutic, PhD. Dissertation, Drew University Madison (NJ) 2005.

Nausner, Michael, Kulturelle Grenzerfahrung und die methodistische Konnexio, in: DERs. (Hg.), Kirchliches Leben in methodistischer Tradition. Perspektiven aus drei Kontinenten, RThS 5 , Göttingen 2010, 273-295.

Nausner, Michael, Postkoloniale Theologien, in: VF 57 (2012), 117-131. 
Nehring, Andreas/Simon Tielisch (Hg.), Postkoloniale Theologien.

Bibelhermeneutische und kulturwissenschaftliche Beiträge, Stuttgart 2013.

Nehring, Andreas/Simon Wiesgickl (Hg.), Postkoloniale Theologien II.

Perspektiven aus dem deutschsprachigen Raum, Stuttgart 2017.

Nolte, MaIK, Des Kaisers Kanonier, in: http://www.taz.de/!5082648/.

Polak, Regina, Migration und Katholizität, in: Dies./Wolfram Reiss (Hg.), Religion im Wandel. Transformation religiöser Gemeinschaften in Europa durch Migration, Interdisziplinäre Perspektiven, Religion and Transformation in Contemporary European Society 9, Wien 2015, 229-292.

polylog 4 (1999): Frau und Kultur. Kolonisierung von Differenz, in: http://www.polylog .net/aktuelles-heft/polylog-4polylog 8 (2001): Hybridität, in: http://www.polylog .net/aktuelles-heft/polylog-8/.

Rosa, Hartmut, Resonanz. Eine Soziologie der Weltbeziehung, Berlin 2016.

SAID, EDWARD W., Orientalism, New York 1978.

SEN, AmARTYA, Identity and Violence. The Illusion of Destiny, London/New York 2013.

Waldenfels, Bernhard, Grundmotive einer Phänomenologie des Fremden, Frankfurt 2006. 